\title{
Metodologia do emprego apoiado: estado atual e caminhos futuros da produção científica
}

Este estudo descreve e analisa as principais características bibliométricas da produção científica sobre a metodologia do Emprego Apoiado para Pessoas com Deficiência, na base de dados web of science. A característica da pesquisa é qualiquantitativa e, pois compreende e explica e enumera e mede. Foram levantados preliminarmente 1.448 trabalhos publicados. O refino da pesquisa levou a uma nova análise de 80 publicações. Emprego Apoiado é tradução para português da expressão Supported Employment, metodologia que surgiu nos Estados Unidos nos anos 80. Pela originalidade do tema, que demonstra as transformações contemporâneas nas relações de trabalho, esta pesquisa introduz e difunde o conhecimento sobre o assunto na área de estudos organizacionais. Conclui-se que há oportunidades de desenvolvimento de futuras pesquisas no campo da Administração e, em especial, relacionadas ao trabalho e organizações.

Palavras-chave: Emprego apoiado; Pessoa com deficiência; Análise bibliométrica.

\section{Supported employment methodology: current state and future paths of scientific production}

This study describes and analyzes the major bibliometric characteristics of scientific literature on the Employment Supported methodology for People with Disabilities, the web of science database. The feature of the research is quantitative, it enumerates and measures, and qualitative because it understands and explains. They were preliminarily raised 1,448 published works. The refining of the research led to a new analysis of 80 publications. Supported Employment is translation into Portuguese of Supported Employment expression, methodology that emerged in the United States in the 80 For the originality of the theme, demonstrating the contemporary changes in labor relations, this research introduces and disseminates knowledge on the subject in the study area organizational. We conclude that there is further research development opportunities in the field of Administration and, in particular, related to work and organizations.

Keywords: Supported employment. People with disabilities. Bibliometric analysis.

\section{Topic: Recursos Humanos}

Reviewed anonymously in the process of blind peer
Received: 07/05/2017

Approved: 08/07/2017
Fernando Vidoi Barboza

Pontifícia Universidade Católica de São Paulo, Brasil http://lattes.cnpq.br/6223376428612257

\section{fbarboza@sp.gov.br}

Belmiro do Nascimento João

Pontifícia Universidade Católica de São Paulo, Brasil http://lattes.cnpq.br/5455434263444485

http://orcid.org/0000-0002-7994-9934

bjoao@pucsp.br
Referencing this:

BARBOZA, F. V.; JOÃO, B. N.. Metodologia do emprego apoiado: estado atual e caminhos futuros da produção científica. Revista Brasileira de Administração Científica, v.8, n.2, p.69-82, 2017. DOI: http://doi.org/10.6008/SPC2179-684X.2017.002.0005

DOI: 10.6008/SPC2179-684X.2017.002.0005 


\section{INTRODUÇÃO}

A Constituição Federal de 1988 (CF/88), nos incisos do art. 7ํ, proíbe diversos tipos de preconceito, seja por questões de cor, gênero, estado civil e especificamente o inciso XXXI proibir qualquer ato discriminatório no tocante a salário e critérios de admissão do trabalhador com deficiência (BRASIL, 1988). Segundo Bezerra et al. (2012), não há igualdade de oportunidade no mercado do trabalho. A desigualdade se faz presente e prejudica diversos segmentos da população. Preconceito e discriminação são as respostas para que milhares de pessoas produtivas e com talento estejam fora do mercado de trabalho (GOFFMAN,1988)

De acordo com o Instituto Brasileiro de Geografia e Estatística (IBGE), dados do censo de 2010 mostram que $23,9 \%$ da população brasileira, ou seja, 45,6 milhões de pessoas apresentam algum tipo de deficiência - visual, auditiva, motora, mental (saúde mental) ou intelectual. Deste montante, 11,3 milhões são considerados em idade economicamente ativa, porém, somente quatro milhões possuem ensino fundamental, médio ou nível superior (IBGE, 2012).

Por outro lado, o Brasil tem avançado na promoção dos direitos das pessoas com deficiência (PcD) por meio de políticas públicas que buscam valorizar a pessoa como cidadã, respeitando suas características e especificidades. Um dos frutos desta política foi a promulgação da Lei n-8.213/91, mais conhecida como Lei de Cotas (BRASIL, 1991). O art. 93 obriga as empresas com 100 ou mais empregados a preencher entre $2 \%$ a $5 \%$ dos seus cargos com PcD habilitadas ou reabilitadas. Segundo dados do Ministério do Trabalho e Emprego (MTE), em 2014 havia 381 mil pessoas (MTE, 2011) com algum tipo de deficiência trabalhando com registro na Carteira de Trabalho e Previdência Social (CTPS).

Segundo a Lei 13.146/2015 (BRASIL, 2015) mais conhecida como Lei Brasileira de Inclusão (LBI), que entrou em vigor em janeiro de 2016, traz mudanças importantes em diversos campos e principalmente amplia o conceito de PcD. Esta lei conceitua deficiência a pessoa que tem algum tipo de impedimento de longo prazo. Este impedimento pode ser de natureza física, mental, intelectual ou sensorial, o qual, em interação com uma ou mais barreiras, pode obstruir sua participação plena e efetiva na sociedade em igualdade de condições com as demais pessoas.

Especificamente, esta pesquisa tratará da metodologia chamada Emprego Apoiado (EA) para PcD no mercado de trabalho. Esta metodologia é desconhecida na literatura científica brasileira, pois na pesquisa realizada, em abril de 2016, no banco de dados da Scientific Periodicals Electronic Library (SPELL) - um dos principais sistemas de indexação, pesquisa e disponibilização da produção científica nas áreas de Administração, Contabilidade e Turismo - não foi encontrada nenhuma publicação.

De acordo com Betti (2011), o EA é uma alternativa de inclusão da pessoa com deficiência no mercado de trabalho auxiliando os empregadores a encontrar candidatos certos para preencher suas ofertas de trabalho colaborando com a diversidade em sua mão de obra. Neste contexto, o EA constrói a diversidade no local de trabalho ajudando as PcD obter e manter um emprego competitivo no mercado de trabalho (SOUSA, 2014). 
Diante disto, o escopo desta pesquisa, utilizando-se de recursos metodológicos da análise bibliométrica, foi o de descrever e analisar a produção científica sobre EA, no portal da Coordenação de Aperfeiçoamento de Pessoal de Nível Superior (CAPES), na base de dados web of science (WoS). Espera-se que o resultado dê a oportunidade de ampliar e disseminar o conhecimento sobre o assunto bem como ampliar as possibilidades de pesquisa sobre o tema. A fim de aprofundar o tema em análise, este artigo foi estruturado em cinco seções: Introdução, Contextualização do tema, Emprego Apoiado, Metodologia e Análise das publicações, Considerações Finais e Referências.

\section{Contextualização do tema}

A desigualdade de oportunidade no mundo do trabalho, ainda hoje, se faz presente e prejudica diversos segmentos da população, seja por questões de etnia, cor, gênero, orientação sexual ou religiosa, entre outras. Preconceito e discriminação são as respostas para que milhares de pessoas com talento e produtivas estejam fora do mercado de trabalho (CLEMENTE, 2015; ROSA, 2014; GROSSI et al., 2013; SANTOS, 2001). A presente pesquisa está justificada na sua relevância social, pois visa concretizar do art. 5ㅇ, da CF que determina que todos são iguais perante a lei, sem distinção de qualquer natureza. 0 art. 23 da Declaração Universal dos Direitos Humanos, das Nações Unidas, estabelece que "toda pessoa tem direito ao trabalho, à liberdade de escolha de emprego, às condições justas e favoráveis de trabalho e à proteção contra o desemprego".

Neste milênio, as PcD representam $10 \%$ da população mundial, equivalentes a aproximadamente um bilhão de pessoas, vivendo com alguma deficiência, segundo a Organização Mundial da Saúde (OMS, 2012). Diante de tal cenário, são necessárias políticas públicas com objetivo de dar qualidade de vida e pleno exercício da cidadania para este extrato da população. Organismos internacionais, como a Organização das Nações Unidas (ONU) e a Organização Mundial da Saúde (OMS) reconhecem que a deficiência faz parte da condição humana. Ações adotadas por estes organismos são de exemplo que norteiam programas de governo de diversos países.

Em 2006, a ONU adota a Convenção sobre os Direitos das Pessoas com Deficiência. No Brasil, tal Convenção foi ratificada através do Decreto Legislativo 186 (BRASIL, 2008). Esta Convenção considera PcD aquelas que têm impedimento de longo prazo de natureza física, mental, intelectual ou sensorial, os quais, em interação com diversas barreiras, podem obstruir sua participação plena e efetiva na sociedade em igualdade de condições com as demais pessoas.

No tocante ao trabalho, o artigo 27 da Convenção (BRASIL, 2008) assegura a liberdade de escolha de trabalho, adaptação física e atitudinal dos locais de trabalho, formação profissional, justo salário em condição de igualdade com qualquer outro cidadão, condições seguras e saudáveis de trabalho, sindicalização, garantia de livre iniciativa no trabalho autônomo, empresarial ou cooperativado, ações afirmativas de promoção de acesso ao emprego privado ou público, garantia de progressão profissional e preservação do emprego, habilitação e reabilitação profissional, proteção contra o trabalho forçado ou escravo, etc. 
Em 2011, a OMS publica o Relatório Mundial sobre a Deficiência (OMS, 2012). Este relatório afirma que nos próximos anos a deficiência será uma preocupação ainda maior - porque sua incidência tende a aumentar por causa do envelhecimento das populações e pelo risco maior de deficiência na população de mais idade, bem como ao aumento global de doenças crônicas como diabetes, doenças cardiovasculares, câncer e distúrbios mentais.

O Relatório faz recomendações para iniciativas nos níveis local, nacional e internacional, sugere ações para governos, organizações da sociedade civil e organizações de PcD, para criar ambientes facilitadores, desenvolver serviços de suporte e reabilitação, garantir uma adequada proteção social, criar programas e políticas de inclusão, e fazer cumprir as normas e a legislação, tanto existentes como novas, para o benefício das PcD e da comunidade como um todo. O Relatório alerta que as PcD devem estar no centro de tais esforços.

É interessante observar que tanto a Convenção da ONU (BRASIL, 2008) e o Relatório da OMS (2012) trabalham o conceito de que "a deficiência faz parte da condição humana". Os dois instrumentos também dedicaram capítulos específicos sobre o Direito ao Trabalho das PCD. No Brasil, a Lei no8.213/91 (BRASIL, 1991) define que toda empresa com 100 ou mais empregados devem preencher entre 2 e $5 \%$ de suas vagas com trabalhadores que tenham algum tipo de deficiência. As empresas que possuem de 100 a 200 empregados devem reservar, obrigatoriamente, 2\% de suas vagas para PcD; entre 201 e 500 empregados, 3\%; entre 501 e 1.000 empregados, 4\%; empresas com mais de 1.001 empregados, 5\% das suas vagas. Segundo dados da Relação Anual de Informações Sociais (RAIS), em 2014 havia cerca de 381 mil PcD formalmente empregadas no Brasil (RAIS, 2015).

Se todas as empresas do país cumprissem a Lei de Cotas (BRASIL, 1991), mais de 900 mil PcD estariam empregadas. Porém, a baixa escolaridade e a falta de qualificação profissional são apontadas como as principais causas da não contratação. Há também, em alguns casos, a necessidade de adaptação na estrutura física das organizações, para que os espaços possam ser adequados ao trabalho e ao deslocamento dos profissionais.

Por outro lado, para mitigar alguns problemas, diversos programas de governos vem sendo adotados em todos os níveis de governo. Em 2011, por meio do Decreto 7.612, o Governo Federal, institui o Plano Nacional dos Direitos da Pessoa com Deficiência - Plano Viver sem Limite (BRASIL, 2011). As ações deste programa são articuladas em parceria com a União, o Distrito Federal, Estados e Municípios. A qualificação profissional, a inclusão, bem como o acesso à tecnologia são alguns aspectos desta política pública. Diante deste cenário, torna-se importante estudar a visão empresarial das práticas adotadas na procura, admissão, integração e manutenção destes profissionais nas organizações.

\section{REVISÃO TEÓRICA}

O termo Supported Employment, que na versão em português foi traduzida como Emprego Apoiado, surgiu nos Estados Unidos no final dos anos 70. Segundo Sassaki (2014a), inicialmente esta metodologia tinha como objetivo resolver o problema da inclusão no mercado de trabalho das pessoas com deficiência 
intelectual. Destaca-se a experiência realizada na Universidade de Virgínia na transição dos estudantes do ensino especial para o cenário do trabalho com a utilização de novas técnicas de integração profissional em ambiente laboral. Segundo Ornelas (2010), a metodologia se apresentava em três serviços básicos: colocação direta no mercado de trabalho, a formação no ambiente de trabalho e o suporte continuado dentro e fora da empresa.

De acordo com Sassaki (2014b), os resultados positivos dos projetos iniciais influenciaram diretamente na criação de legislação específica garantindo verbas e suportes para programas de Emprego Apoiado no país e repercutindo diretamente no número de pessoas atuando nesta modalidade (de 9.000 em 1986 para 72.000 em 1990). O termo Emprego Apoiado, em português, é o resultado da tradução do termo Supported Employment. Em consulta ao dicionário Oxford, Supported origina-se do verbo support, que quer significa 'apoiar; dar respaldo a', enquanto o vocábulo Employment é traduzido como 'emprego, trabalho'. A partícula ed possui no inglês, de tempo verbal passado (apoio = apoiado; respaldo = respaldado), chegamos ao termo 'Emprego Apoiado'.

O paradigma até então era capacitar, isto é, treinar e desenvolver habilidades, geralmente por meio de oficinas protegidas de trabalho, e depois fazer a colocação no mercado de trabalho. Isto é, a pessoa desenvolvia competências em uma determinada profissão e estando apta ao trabalho era direcionada ao mercado de trabalho. De acordo com Betti (2011), o EA muda totalmente este paradigma, isto é, valoriza a importância da inversão do "treinar-colocar" para "colocar-treinar" como uma forma de se contrapor ao que era feito nas oficinas protegidas de trabalho. A metodologia do EA tem três fases, a saber:

Descoberta do Perfil Vocacional: através de um profissional, chamado técnico de EA, faz o levantamento dos interesses e necessidades da pessoa com deficiência. Neste paradigma, a pessoa torna-se o protagonista de sua história. Nesta fase, é feita a abordagem com todas as pessoas que se relacionam com a PcD. Com o objetivo de detectar possíveis barreiras de acessibilidade, é levantado aspectos da infraestrutura do entorno da moradia;

Desenvolvimento de Emprego: nesta fase, são realizadas pesquisa e abordagem com empresas para avaliar possíveis atividades aderentes ao perfil vocacional. Para tanto, verifica-se a compatibilidade entre o perfil da pessoa com a atividade que será executada. Faz-se análise da disponibilidade e adequação de apoios naturais com o objetivo de eliminação de barreiras. $O$ resultado deste processo é a criação de uma vaga customizada que atenda tanto as necessidades da empresa como do futuro empregado. Em se havendo a contratação, realiza-se um Plano Individual de Treinamento e Inclusão Social sob a responsabilidade do Consultor de EA. Aqui está a principal diferença com outras metodologias convencionais de inclusão. A formação e treinamento são realizados na empresa, proporcionando conhecimento prático necessário para realizar as atividades;

Acompanhamento pós-colocação: é realizado o acompanhamento do Plano Individual de Treinamento verificando se as estratégias e os apoios naturais estão adequados. Aos poucos, o Consultor de EA vai se distanciando à medida que a empresa e o empregado vão ganhando confiança e independência. Paulatinamente, o acompanhamento é feito a distância tendo por objetivo garantir a qualidade da inclusão, intervindo somente em situações mais desafiadoras e auxiliando no desenvolvimento da carreira profissional do empregado. (BETTI, 2011)

Segundo Gonzalez et al. (2014), é fundamental destacar, em todo o processo, o papel do Consultor de EA. Este profissional é responsável na avaliação da pessoa, na colocação no posto de trabalho, na customização do treinamento e na avaliação contínua. O EA surge para ser uma alternativa viável de acesso ao trabalho para as pessoas, até então, simplesmente excluídas ou atendidas precariamente pelos programas 
existentes. Ao enfatizar que é a situação que gera uma incapacidade mais significativa para se conseguir um trabalho e não o indivíduo que possui uma deficiência, o EA revela seu compromisso com uma visão baseada no modelo social da deficiência, ou seja, a incapacidade não está na pessoa, e sim no meio ambiente (NUERNBERG, 2015).

Segundo Bampi et al. (2010), o modelo social é um contraponto do modelo médico de deficiência que entende a lesão e a limitação física a causa da desigualdade social e das desvantagens vivenciadas pelos deficientes. Este modelo ignora o papel da sociedade na sua opressão e marginalização da PcD. O paradigma deste modelo é que a PCD tem de se adaptar à sociedade.

Por outro lado, o modelo social entende que a incapacidade é fruto das desvantagens ou restrições provocadas pela organização social contemporânea que pouco ou nada considera aqueles que possuem lesões físicas e os exclui das principais atividades da sociedade. Em resumo, o paradigma do modelo social é que a deficiência e a incapacidade não devem ser entendidas como um problema individual, mas uma questão da vida em sociedade, o que transfere a responsabilidade pelas desvantagens das limitações corporais do indivíduo para a incompetência da sociedade em prever e se ajustar à diversidade (BAMPI et al., 2010).

Deste modo, a ênfase deve recair na implementação de um bom plano de apoio e no enfrentamento das barreiras ambientais que prejudicam a participação plena da pessoa no trabalho e em outras áreas da vida. Mais do que apenas uma nova metodologia de trabalho, o EA solicita uma mudança estrutural na forma de pensar e conceber o acesso ao trabalho de pessoas em situação de incapacidade. O EA estabelece que, se é uma situação de incapacidade que precisa ser modificada, isso só ocorrerá na medida em que se entenda o papel e forma de interação da sociedade em relação à pessoa com deficiência.

\section{METODOLOGIA}

A metodologia adotada neste estudo é de natureza bibliométrica, pois se analisou a produção científica sobre EA presente nos principais periódicos na base do WoS. Este método se trata de uma revisão de literatura sobre as teorias fundamentais que embasam o trabalho científico (PIZZANI et al., 2012). De acordo com Mueller (2013), o levantamento bibliográfico têm os seguintes objetivos: mapeamento de autorias e coautorias, colaboração e redes; avaliação e descrição da literatura, impacto e indicadores; produção e produtividade, visibilidade de autores e instituições e estudos de citação e co-citação. Ressalvase que há limitações, segundo afirmativa de Tasca et al. (2010): a relação do pesquisador com as delimitações por ele definidas para a pesquisa é influenciada pelo contexto no qual o pesquisador está inserido e pela disponibilidade de acesso aos meios de divulgação de pesquisas.

\section{Busca sistemática da literatura}

O levantamento e a análise deste estudo foram realizados em abril de 2016, a partir das bases de dados da WoS que disponibiliza acesso ao conteúdo integral de pesquisa científica mais importante do mundo, incluindo revistas acadêmicas, livros, anais, conjuntos de dados publicados e patentes. Inicialmente, 
para a coleta de dados acerca do assunto, foi introduzida no campo de pesquisa básica a expressão 'Supported Employment'. A pesquisa limitou-se a publicações até o ano de 2015. Com estes parâmetros, encontrou-se 1.448 publicações indexadas.

Com estes dados, foi possível organizar os dados bibliométricos referentes às publicações localizadas. Através da funcionalidade 'Análise de dados', foi possível a criação de tabelas e figuras para representar indicadores de frequência das publicações por ano; principais autores, principais periódicos; instituições e países mais produtivos.

Com os resultados apresentados, foi realizada nova rodada na pesquisa para alinhar as publicações ao escopo da pesquisa, isto é, buscar apenas publicações na área de conhecimento considerada relevante para a pesquisa, no caso as Ciências Sociais Aplicadas, tais como: Behavioral Sciences, Business, Law, Psychology Social, Management, Public Administration, Social Issues, Economics, Social Work e Sociology. Após o refinamento dos critérios foram totalizadas 80 publicações. Através da ferramenta EndNote, disponível no ambiente do web of science, foi possível exportar os dados das 80 publicações e verificar, autores mais produtivos, textos mais citados, a frequência de palavras-chave que mais apareceram. A seguir, os resultados são apresentados na forma de tabelas e gráfico, cuja finalidade é facilitar a visualização dos dados levantados.

\section{RESULTADOS E DISCUSSÃO}

Nesta etapa, são apresentados e discutidos os principais resultados oriundos da busca sistemática e da análise descritiva da literatura. A partir da análise bibliométrica, foram encontradas, indexadas à base de dados WoS, 1448 publicações. Estes artigos foram escritos por 2.606 autores com vínculo em 816 instituições de 27 países; foram publicados em 52 periódicos (fontes de publicação).

Inicialmente, o objetivo foi encontrar o ano das primeiras publicações e evolução quantitativa de publicações ao longo dos anos. O início das publicações data de 1987 com quatro artigos, podendo considerar dois deles, publicados no Journal of Rehabilitation, como seminais. O primeiro artigo, com o título Supported Employment: An Interagency Funding Model for Persons with Severe Disabilities, desenvolvida por Hill et al. (1987) apresenta a metodologia do EA para pessoas com deficiência, fazendo distinção entre os serviços de preparação de pré-emprego convencional e serviços pós-emprego, mostrando impactos do programa de serviço em adultos com deficiência. $\mathrm{O}$ segundo artigo, Supported Employment - Challenge and Opportunity for Sheltered Workshops, escrito por Whitehead (1987) apresenta o surgimento do EA, sua implementação e vantagens sobre as oficinas protegidas de trabalho.

No que se refere a evolução das publicações por ano, é possível conferir na figura 1, a frequência das mesmas no período que compreende os anos de 1987 e 2015. Entre 1990 e 2008, a quantidade de publicações segue o ritmo progressivo, até que a produção salta de 74 trabalhos em 2008 para 100 em 2009. Em 2010, são registradas 86 publicações e atinge o ápice em 2014, com 123 publicações. 


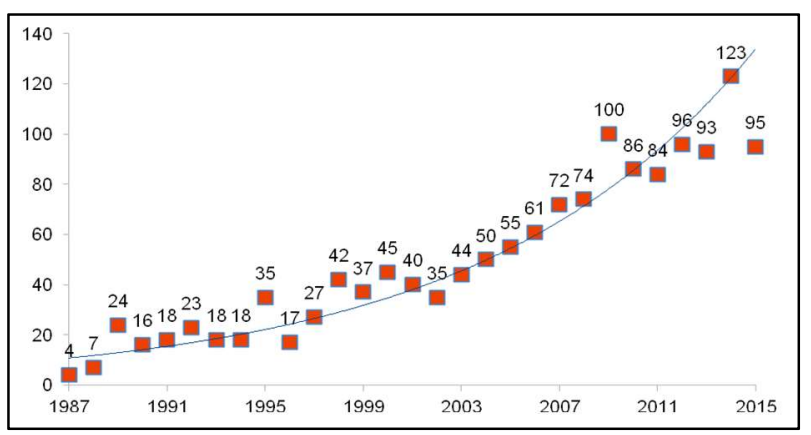

Figura 1: Publicações entre os anos de 1987 e 2015.

Progressivamente, a cada ano nota-se uma tendência de aumento das publicações demonstrando o interesse de pesquisadores sobre a temática. Posteriormente, foi feito o levantamento dos autores que apresentaram publicações, limitando-se a 25 , relacionadas ao EA como se pode visualizar na tabela 1, que traz, literalmente, a 'relação de autores e quantidade de publicações.

Tabela 1: Relação de autores e quantidade de publicações.

\begin{tabular}{|l|c|}
\hline \multicolumn{1}{|c|}{ AUTOR } & QTDE \\
\hline Drake R.E. & 109 \\
\hline Bond G.R. & 92 \\
\hline Becker D.R. & 63 \\
\hline Mueser K.T. & 47 \\
\hline Waghorn G. & 36 \\
\hline Wehman PP. & 33 \\
\hline Mcgurk S.R. & 25 \\
\hline
\end{tabular}

Com 109 publicações, Robert E. Drake, Doutor pela Duke University, é diretor do programa sobre Política de Saúde e Clínica Prática da Faculdade de Medicina Geisel do Instituto Dartmouth. É professor de Psiquiatria e Medicina da Família e da Comunidade no mesmo Instituto onde se dedica a pesquisas na conceituação, desenvolvimento, avaliação e disseminação de práticas baseadas em evidências em EA. Logo a seguir, parceiro de Drake em mais de 80 trabalhos, destaca-se Gary R. Bond, professor de Psiquiatria na mesma instituição, pesquisa o desenvolvimento, avaliação, implementação e divulgação internacional de práticas de reabilitação psiquiátrica baseadas em evidências focada no modelo de EA. Desse modo o The Dartmouth Institute for Health Policy and Clinical Practice (TDI) é uma referência mundial em pesquisa sobre o tema. Foi realizado o levantamento dos principais veículos de comunicação na academia (tabela 2).

Tabela 2: Quantidade de publicações no periódico.

\begin{tabular}{|l|c|}
\hline \multicolumn{1}{|c|}{ PUBLICAÇÃO } & QTDE \\
\hline Psychiatric Rehabilitation Journal $^{\circledR}$ & 127 \\
\hline Psychiatric Services $_{|c|}$ & 100 \\
\hline Journal of Rehabilitation & 67 \\
\hline Work A Journal of Prevention Assessment Rehabilitation & 40 \\
\hline Community Mental Health Journal & 39 \\
\hline Journal of the Association for Persons with Severe Handicaps & 37 \\
\hline Schizophrenia Bulletin & 37 \\
\hline Journal of Intellectual Disability Research & 34 \\
\hline Education and Training in Mental Retardation and Developmental Disabilities & 29 \\
\hline Journal of Applied Research in Intellectual Disabilities & 25 \\
\hline
\end{tabular}

O Psychiatric Rehabilitation Journal ${ }^{\circ}$, índice de impacto JCR =1,169, foi o veículo de publicação com maior incidência de artigos relacionados ao tema, com 127 artigos publicados. Tem por editores Judith A. 
Cook e Kim T. Mueser. A primeira é professora de psiquiatria e diretora do Center on Mental Health Services Research and Policy vinculado ao Departamento de Psiquiatria da Faculdade de Medicina da Universidade de Illinois, Chicago.

O segundo é diretor executivo do Center for Psychiatric Rehabilitation (CPR) e professor do Departmento de Terapia Ocupacional do Sargent College em Boston. O Journal é associado a American Psychological Association (APA) e é o journal oficial da Psychiatric Rehabilitation Association e da Universidade de Boston (Boston University Center for Psychiatric Rehabilitation). Este Journal publica artigos relacionados com a reabilitação, tratamento psicossocial e recuperação de pessoas com transtornos mentais graves. O journal oferece investigações de pesquisa clínica e básica; formulações teóricas; revisões de literatura; estudos de caso; discussões sobre questões de política pública e resenhas de livros.

Com 40 publicações, a Work A Journal of Prevention Assessment Rehabilitation é ligada à Canadian Institute for the Relief of Pain and Disability, é uma revista interdisciplinar que publica artigos com questões temáticas que abrangem uma vasta gama de tópicos como considerações ergonômicas com crianças, jovens e estudantes, os desafios que enfrentam a força de trabalho em envelhecimento, violência no trabalho, gestão de deficiências, artistas, avaliações de produtos ergonômicos, e a consciência dos determinantes políticos, culturais e ambientais da saúde relacionados com o trabalho. Não há publicações expressivas em publicações voltadas para os estudos organizacionais, pois das 1.448 publicações, somente 40 , ou seja, $2,7 \%$ foram publicadas especificamente em veículo de publicação voltado ao trabalho. A maioria das publicações está vinculada à área da saúde.

Realizou-se o levantamento dos países onde se concentram as publicações desses artigos, ou seja, os países que mais têm se dedicado ao estudo do tema. Neste item, ressalvada a possibilidade de uma publicação ter mais de uma incidência entre países, observa-se que os Estados Unidos da América (EUA) lideram o ranking com 891 publicações (tabela 3), seguido da Inglaterra com 122 publicações, Austrália, 111 e Canadá com 108. A maior incidência de publicações nos EUA é explicada pelo fato da concepção do modelo de EA ser originaria deste país. Portanto, há oportunidade de expansão dos estudos relacionados ao tema para outros países e culturas.

Tabela 3: Quantidade de publicações, por país.

\begin{tabular}{|l|c|}
\hline PAíS & QTDE \\
\hline EUA & 891 \\
\hline Inglaterra & 122 \\
\hline Austrália & 111 \\
\hline Canadá & 108 \\
\hline Alemanha & 51 \\
\hline Holanda & 47 \\
\hline Suiça & 34 \\
\hline Itália & 29 \\
\hline Taiwan & 27 \\
\hline China & 25 \\
\hline Espanha & 20 \\
\hline
\end{tabular}

Quanto ao idioma de publicação, dados expostos na tabela 4, destaca-se que 1.392 foram realizadas na língua inglesa, 36 em alemão e 15 em francês. Única publicação em idioma português, Bonadio et al. 
(2013) afirma que, no contexto brasileiro, não há política pública de EA, limitando-se a ações de cooperativismo.

Tabela 1: Quantidade de publicações por idioma.

\begin{tabular}{|l|c|}
\hline IDIOMA & QTDE \\
\hline Inglês & 1392 \\
\hline Alemão & 36 \\
\hline Francês & 15 \\
\hline Italiano & 4 \\
\hline Espanhol & 3 \\
\hline
\end{tabular}

Em relação às instituições, a Dartmouth College, através de sua Faculdade de Medicina Geisel, com 166 artigos, conforme se nota na tabela 5, foi a instituição que se destacou pela quantidade de publicações. Observa-se, também que da lista das dez primeiras instituições listadas, somente uma, University of London, fica fora dos EUA.

Tabela 5: Quantidade de publicações por Instituição.

\begin{tabular}{|l|c|}
\hline \multicolumn{1}{|c|}{ INSTITUIÇÃO } & QTDE \\
\hline Dartmouth College & 166 \\
\hline Indiana University System & 90 \\
\hline Indiana University/ Purdue University Indianapolis & 80 \\
\hline Yale University & 59 \\
\hline University of California System & 57 \\
\hline Virginia Commonwealth University & 56 \\
\hline Boston University & 54 \\
\hline University of London & 52 \\
\hline University System of Maryland & 49 \\
\hline University of Illinois System & 44 \\
\hline
\end{tabular}

A tabela 6, que traz em seu bojo a quantidade de publicações por área do conhecimento, elenca as principais áreas de conhecimento que tiveram trabalho publicado, obtendo-se um total de 2308 publicações. A maioria delas é na área de Saúde, e somente 20 publicações estão enquadradas no escopo desta pesquisa, isto é, área organizacional.

Tabela 2: Quantidade de publicações, por área de conhecimento.

\begin{tabular}{|l|l|}
\hline ÁREA & QTDE \\
\hline Psychiatry & 670 \\
\hline Rehabilitation & 636 \\
\hline Public Environmental Occupational Health & 232 \\
\hline Health Care Sciences Services & 201 \\
\hline Psychology & 174 \\
\hline Education Educational Research & 162 \\
\hline Neurosciences Neurology & 134 \\
\hline Genetics Heredity & 36 \\
\hline Social Issues & 22 \\
\hline Social Sciences other Topics & 21 \\
\hline Social Work & 20 \\
\hline
\end{tabular}

Analisando os resultados apresentados, foi realizado o refinamento na busca, com objetivo de alinhar o tipo de publicação com o escopo da pesquisa, isto é, buscar apenas publicações na área de conhecimento considerada relevante para a pesquisa, no caso as Ciências Sociais Aplicadas, tais como: Behavioral Sciences, Business, Law, Social Psychology, Management, Public Administration, Social Issues, Economics, Social Work e Sociology. Após aplicação do filtro, foram totalizadas 80 publicações. 
Realizou-se análise detalhada destas publicações envolvendo com enfoque no resumo geral, palavras-chave, incidência de citações das publicações, entre outras informações. Essas informações são relevantes por se reconhecer que o valor de cada publicação é determinado por quem as usa e pela quantidade de uso que se faz dela (COSTA FILHO, 2009). A frequência das citações reflete o valor de um periódico e o uso feito dele. Assim, no caso de uma publicação importante, o índice de citações tem um valor quantitativo, porque ele pode ajudar o pesquisador a medir a influência do artigo - isto é, seu fator de impacto (COSTA FILHO, 2009). Os 80 artigos tiveram um total de 1.042 citações.

O Journal of Occupational Rehabilitation, fator de impacto JCR=2,159, foi o periódico com maior incidência de artigos relacionados ao tema, com 16 publicações (tabela 7). Esse Journal publica artigos sobre a reabilitação do trabalhador com deficiência. A revista oferece investigações de pesquisa clínica e básica; formulações teóricas; revisões de literatura; estudos de caso; discussões sobre questões de política pública e resenhas de livros. Nota-se que tal publicação, apesar de tratar do assunto Trabalho é voltada para a área da Saúde. Não há publicações expressivas em periódicos voltadas para os estudos organizacionais. A área de Reabilitação apresentou 27 (tabela 8) publicações e em relação a área do conhecimento.

Tabela 3: Quantidade de publicações por Periódico.

\begin{tabular}{|l|c|}
\hline \multicolumn{1}{|c|}{ PUBLICAÇÃO } & QTDE \\
\hline Journal of Occupational Rehabilitation & 16 \\
\hline Disability Society & 11 \\
\hline Health Social Care in The Community & 5 \\
\hline Research on Social Work Practice & 5 \\
\hline Social Work & 4 \\
\hline American Journal of Evaluation & 3 \\
\hline
\end{tabular}

Tabela 4: Quantidade de publicação por área do conhecimento.

\begin{tabular}{|l|c|}
\hline \multicolumn{1}{|c|}{ ÁREA } & QTDE \\
\hline Rehabilitation & 27 \\
\hline Social Issues & 21 \\
\hline Social Sciences other Topics & 20 \\
\hline Social Work & 20 \\
\hline Business Economics & 15 \\
\hline Psychology & 9 \\
\hline Public Environmental Occupational Health & 6 \\
\hline Public Administration & 5 \\
\hline Government Law & 4 \\
\hline Psychiatry & 4 \\
\hline Health Care Sciences Services & 2 \\
\hline \multicolumn{1}{|c|}{ Total } & 133 \\
\hline
\end{tabular}

Ressalta-se que a frequência de publicações foi para 133 (tabela 8). Isto se deve ao fato de um mesmo artigo publicado estar enquadrado em mais de uma área temática. Surpreendentemente, na área de Management não houve publicação encontrada, porém a área mais próxima, Social Work, apresentou 20 publicações. Conclui-se que há um desafio em aproximar o tema na área de ciências sociais aplicadas, especificamente em estudos organizacionais. Dos 80 artigos identificados, foi feito o levantamento dos autores que apresentaram mais de uma publicação relacionada ao EA (tabela 9).

Destaca-se a pesquisadora inglesa Justine Schneider da Universidade de Nottingham, apresentando trabalhos na avaliação mental dos serviços de saúde, os prestadores de cuidados, casas de cuidados, custos e emprego apoiado para pessoas com qualquer tipo de deficiência. Na sequência, Gary R. Bond, já 
mencionado anteriormente, é professor do Instituto Dartmouth. Sua pesquisa se concentra no desenvolvimento, avaliação, implementação e divulgação internacional de práticas de reabilitação psiquiátrica baseadas em evidências, com enfoque no modelo de EA.

Tabela 5 - Quantidade de publicações por autor.

\begin{tabular}{|l|l|}
\hline AUTOR & QTDE \\
\hline Schneider J & 5 \\
\hline Bond GR & 3 \\
\hline Chang YJ & 3 \\
\hline Rogers ES & 3 \\
\hline Secker J & 3 \\
\hline Becker DR & 2 \\
\hline
\end{tabular}

O artigo mais citado, com 207 citações, Fidelity criteria: Development, measurement, and validation, foi publicado em 2003 no American Journal of Evaluation escrito por Mowbray, Holter, Teague \& Bybee (2003). Na tabela 10, pode ser verificada a relação das publicações mais citadas incluindo informações como: título, periódicos, autores, ano de publicação e quantidade de citação.

Tabela 10: Quantidade de citações por título, periódico, autores e ano de publicação.

\begin{tabular}{|c|c|c|c|c|}
\hline TÍTULO & PERIÓDICO & AUTORES & ANO & CITAÇÕES \\
\hline $\begin{array}{l}\text { Fidelity criteria: Development, } \\
\text { measurement, and validation }\end{array}$ & $\begin{array}{l}\text { American Journal of } \\
\text { Evaluation }\end{array}$ & $\begin{array}{l}\text { Mowbray, C. T; Holter, MC; } \\
\text { Teague, GB; Bybee, D }\end{array}$ & 2003 & 207 \\
\hline $\begin{array}{l}\text { Modified work and returns to } \\
\text { work: A review of the literature }\end{array}$ & $\begin{array}{l}\text { Journal of } \\
\text { Occupational } \\
\text { Rehabilitation }\end{array}$ & $\begin{array}{l}\text { Krause, N; Dasinger, LK; } \\
\text { Neuhauser, F }\end{array}$ & 1998 & 175 \\
\hline $\begin{array}{l}\text { Employment outcomes in family- } \\
\text { aided assertive community } \\
\text { treatment }\end{array}$ & $\begin{array}{l}\text { American Journal of } \\
\text { Orthopsychiatry }\end{array}$ & $\begin{array}{l}\text { McFarlane, WR; Dushay, RA; } \\
\text { Deakins, SM; Stastny, P; Lukens, } \\
\text { EP; Toran, J; Link, B }\end{array}$ & 2000 & 67 \\
\hline $\begin{array}{l}\text { A Framework for Measuring } \\
\text { Fidelity of Implementation: A } \\
\text { Foundation for Shared Language } \\
\text { and Accumulation of Knowledge }\end{array}$ & $\begin{array}{l}\text { American Journal of } \\
\text { Evaluation }\end{array}$ & $\begin{array}{l}\text { Century, Jeanne; Rudnick, Mollie; } \\
\text { Freeman, Cassie }\end{array}$ & 2010 & 55 \\
\hline $\begin{array}{l}\text { Strategies for Improving Fidelity } \\
\text { in the National Evidence-Based } \\
\text { Practices Project }\end{array}$ & $\begin{array}{l}\text { Research on Social } \\
\text { Work Practice }\end{array}$ & $\begin{array}{l}\text { Bond, Gary R.; Drake, Robert E.; } \\
\text { McHugo, Gregory J.; Rapp, } \\
\text { Charles A.; Whitley, Rob }\end{array}$ & 2009 & 36 \\
\hline $\begin{array}{l}\text { A Benefit-Cost Analysus if a } \\
\text { Supported Employment model } \\
\text { for persons with psychiatric } \\
\text { disabilities. }\end{array}$ & $\begin{array}{l}\text { Evaluation and } \\
\text { Program Planning }\end{array}$ & $\begin{array}{l}\text { Rogers, ES; Sciarappa, K; } \\
\text { MacDonaldwilson, K; Danley, K }\end{array}$ & 1995 & 31 \\
\hline $\begin{array}{l}\text { Unique issues in assessing work } \\
\text { function among individuals with } \\
\text { psychiatric disabilities }\end{array}$ & \begin{tabular}{|l|} 
Journal of \\
Occupational \\
Rehabilitation \\
\end{tabular} & $\begin{array}{l}\text { MacDonald-Wilson, K; Rogers, } \\
\text { ES; Anthony, WA }\end{array}$ & 2001 & 29 \\
\hline $\begin{array}{l}\text { Employment of individuals with } \\
\text { mental disabilities: Business } \\
\text { response to the ADA'S challenge }\end{array}$ & $\begin{array}{l}\text { Behavioral Sciences \& } \\
\text { The Law }\end{array}$ & Scheid, TL & 1999 & 28 \\
\hline $\begin{array}{l}\text { Recovering from illness or } \\
\text { recovering your life? Implications } \\
\text { of clinical versus social models of } \\
\text { recovery from mental health } \\
\text { problems for employment } \\
\text { support services }\end{array}$ & Disability \& Society & $\begin{array}{l}\text { Secker, J; Membrey, H; Grove, B; } \\
\text { Seebohm, P }\end{array}$ & 2002 & 26 \\
\hline $\begin{array}{l}\text { Place first, then train: An } \\
\text { alternative to the medical model } \\
\text { of psychiatric rehabilitation }\end{array}$ & Social Work & Corrigan, PW; McCracken, SG & 2005 & 25 \\
\hline
\end{tabular}

\section{CONCLUSÕES}

De acordo com os levantamentos efetuados, foi possível obter um panorama da metodologia EA, desde as publicações seminais, que surgiram na década de 1980, às mais recentes publicações. Constatou-se 
que houve poucas produções científicas na área de management, porém vasta utilização em outros campos do conhecimento. Os resultados evidenciam a forte presença de interdisciplinaridade, tendo em vista que pesquisadores de diferentes áreas científicas realizam estudos sobre a temática.

Apesar das limitações da pesquisa, restritas aos artigos publicados em periódicos científicos nas bases de dados WoS, disponíveis no Portal da Capes, o tema é relevante e inédito na área de organizações trazendo reflexões de alternativas possíveis das relações de trabalho, organização e sociedade. Acredita-se que os resultados possam contribuir com pesquisadores brasileiros, pela introdução da temática e pelo possível despertar de interesse para futuras pesquisas que sistematizem as transformações contemporâneas das relações trabalho.

\section{REFERÊNCIAS}

BAMPI, L. N. S.; GUILHEM, D.; ALVES, E. D.. Modelo Social: Uma nova abordagem para o tema deficiência. Revista Latino-Americana de Enfermagem, Ribeirão Preto, v.18, n.4, p.816-823, 2010.

BETTI, A. P.. Emprego Apoiado. São Paulo: AGbook, 2011.

BEZERRA, S. S.; VIEIRA: M. M. F.. Pessoa com Deficiência Intelectual: a nova 'ralé' das organizações do trabalho. Revista de Administração de Empresas, v.52, n.2, 2012.

BONADIO, A. N.; SILVEIRA, C.. Cooperative Work and Vocational Rehabilitation in the Drug Addiction Treatment: opportunities and limitations of current practices. Saúde e Sociedade, São Paulo, v.22, n.1, p.99-108, 2013.

BRASIL. Constituição da República Federativa do Brasil. Brasília: Senado Federal, 1988.

BRASIL. Decreto n.7612 de 17 de novembro de 2011. Institui o Plano Nacional dos Direitos da Pessoa com Deficiência: Plano Viver sem Limite. Brasília: DOU, 2016.

BRASIL. Lei n.13146 de 6 de julho de 2015. Institui a Lei Brasileira de Inclusão da Pessoa com Deficiência (Estatuto da Pessoa com Deficiência) Brasília: DOU, 2016.

BRASIL. Lei n.7853 de 24 de outubro de 1989. Dispõe sobre o apoio às pessoas portadoras de deficiência, sua integração social, sobre a Coordenadoria Nacional para Integração da Pessoa Portadora de Deficiência - Corde institui a tutela jurisdicional de interesses coletivos ou difusos dessas pessoas, disciplina a atuação do Ministério Público, define crimes, e dá outras providências. Brasília: DOU, 1989.

BRASIL. Lei n.8213 de 24 de julho de 1991. Dispõe sobre Planos de Benefícios da Providência Social e dá outras providências. Brasília: DOU, 1991.

CLEMENTE, C. A.. Pessoas com Deficiência: Derrubando mitos e preconceitos. Revista Ciências do Trabalho, n.4, p.139-144, 2015.

COSTA FILHO, A. R.. Dossiê: C\&T - análises sobre a cultura da avaliação na produção acadêmica. Estatística de Citações. Mediações. Revista de Ciências Sociais, v.14, n.1, p.69-100, 2009.
GOFFMAN, E.. Estigma: Notas sobre a manipulação da identidade deteriorada. 4 ed. Rio de Janeiro: Guanabara, 1988.

GONZALEZ, F.; MARTINEZ, V.; SCOTTON, A.. Emprego Apoiado: Uma leitura psicanalítica. São Paulo: AGBook, 2014.

GROSSI, M. P.; SCHENDEILWEIN, I. L.; MASSA, J. M. Discriminação tem gênero no Brasil. GV-executivo, v.12, n.1, 2013.

HILL, M.; HILL, J.; WEHMAN, P.; REVEL, G.; DICKERSON, A.; NOBLE, J.. Supported Employment: An interagency funding model for persons with severe disabilities. Journal of Rehabilitation, v.53, n.3, 1987.

IBGE. Instituto Brasileiro de Geografia e Estatística. Censo Demográfico 2010: Características gerais da população, religião e pessoas com deficiência. Rio de Janeiro: IBGE, 2012.

MOWBRAY, C. T.; HOLTER, M. C.; TEAGUE, G. B.; BYBEE, D. Fidelity Criteria: Development, Measurement, and Validation. American Journal of Evaluation, 2003.

MTE. Ministério do Trabalho e Emprego. Inclusão do Trabalhador Deficiente Cresce Ano a Ano. Brasília: MTE, 2011.

MUELLER, S. P. M.. Estudos Métricos da Informação em Ciência e Tecnologia no Brasil Realizados sobre a Unidade de Análise de Artigos de Periódicos. Liinc em Revista, Rio de Janeiro, v.9, n.1, 2013.

NUERNBERG, A. H.. Os Estudos sobre Deficiência na Educação. Educação \& Sociedade, v.36, n.131, 2015.

OMS. Organização Mundial da Saúde. Relatório Mundial sobre a Deficiência. São Paulo: SEDPcD, 2012.

ORNELAS, J. H.. Os contributos do emprego apoiado para a integração das pessoas com doença mental. Análise Psicológica, v.28, n.3, p.465-478, 2010.

PIZZANI, L.; SILVA, R. C.; BELLO, S. F.; HAYASHI, M. C. P. I.. A arte da Pesquisa Bibliográfica na Busca do Conhecimento. 
Revista Digital de Biblioteconomia e Ciência da Informação, Campinas, v.10, n.1, p.53-66, 2012.

RAIS. Relação Anual de Informações Sociais. Resultados definitivos. Brasília: MT, 2015.

ROSA, A. R.. Relações Raciais e Estudos Organizacionais no Brasil. Revista de Administração Contemporânea, Curitiba, v.18, n.3, p.240-260, 2014. http://doi.org/10.1590/19827849rac20141085

SANTOS, H.. Discriminação Racial no Brasil. In: SEMINÁRIOS REGIONAIS PREPARATÓRIOS PARA A CONFERÊNCIA MUNDIAL CONTRA O RACISMO, DISCRIMINAÇÃO RACIAL, XENOFOBIA E INTOLERÂNCIA CORRELATA. Anais. Brasília: MJ, 2001.
SASSAKI, R. K.. História do Emprego Apoiado no Brasil. Revista Deficiência Intelectual, v.4, n.6, 2014b.

SASSAKI, R. K.. Inclusão laboral mediante emprego apoiado. Revista Nacional de Reabilitação, São Paulo, v.17, n.99, p.12-13, 2014a.

SOUSA, A.. Emprego Apoiado: uma primeira abordagem. Psicología, v.14, n.1, p.73-82, 2014.

TASCA, J. E.; ENSSLIN, L.; ENSSLIN, S. R.; ALVES, M. B. M.. An approach for selecting a theoretical framework for the evaluation of training programs. Journal of European Industrial Training, v.34, n.7, p.631 -655, 2010.

WHITEHEAD, C. W.. Supported employment: Challenge and opportunity for sheltered workshops. Journal of Rehabilitation, v.53, n.3, p.23-28, 1987. 